Journal of Accident and Emergency Medicine 1995 12, 300-301
Correspondence: S.C. Clark, The Transplant Office, Cardiothoracic Centre, Freeman Hospital, Newcastle upon Tyne NE7 7DN, UK

\title{
Bilateral patellar tendon rupture secondary to repeated local steroid injections
}

\author{
S.C. CLARK, M.W. JONES, R.R. CHOUDHURY \& E. SMITH
}

Department of Orthopaedic Surgery, Leicester Royal Infirmary, Infirmary Road, Leicester, UK

\section{SUMMARY}

A case is reported of bilateral patellar tendon rupture in a fit man after a fall. He had a history of repeated local steroid injections into both tendons and histology confirmed steroid-induced changes. The history of repeated local steroid administration has to be implicated as the cause of this extremely rare injury in this patient, which can also be associated with hyperparathyroidism, systemic lupus erythematosus, diabetes and rheumatoid arthritis. All doctors performing repeated local steroid injections into the patellar tendon should be aware of the possible dangers of inducing tendon rupture and should ensure that the steroids are not delivered into its substance.

Key words: patellar tendons, ruptures, steroid injections

\section{CASE REPORT}

A 43-year-old man presented to the accident and emergency (A\&E) department after tripping on a manhole cover and twisting both knees as he fell. He was subsequently unable to stand.

He reported 6 years of bilateral anterior knee pain, which he had attributed to minor sporting injuries. He had received approximately six steroid injections into each patellar tendon, which he had found to be beneficial.

On examination, deficits in both patellar tendons were palpable and active extension was absent in both knees. No other abnormal findings were noted. Plain radiographs showed superior displacement of both patellae with no degenerative changes. Surgery showed that rupture had occurred through the substance of each tendon and direct repair was undertaken, protected by tension band wiring.
Histological examination of the patellar tendon specimens confirmed non-specific changes of scanty fibroblasts and blood vessels consistent with degeneration secondary to local steroid administration.

Post-operatively he made a good recovery and on follow-up at 6 months he continued to make good progress.

\section{DIsCussion}

Rupture of the patellar tendon is unusual, but is an important disorder that may present to the A\&E doctor.

Because of the great tensile strength of normal tendons, rupture through their substance is usually associated with a degenerative or pathological process. Normal patellar tendons do not rupture in their substance, but usually at the musculotendinous or osseotendinous junction at the lower pole of the patella.'

Bilateral rupture of the patellar tendon in healthy subjects secondary to trauma is exceptionally rare, ${ }^{2}$ but has been more commonly reported in conjunction with rheumatoid arthritis, ${ }^{3}$ systemic lupus erythematosus, ${ }^{4}$ and arteriosclerosis. Other factors such as diabetes mellitus, hyperparathyroidism $^{5}$ and renal failure ${ }^{6}$ have also been cited. These disorders were sought and excluded in this patient, but must be borne in mind when assessing other patients with suspected patellar tendon rupture.

Both local and systemic steroid administration have been implicated in unilateral rupture, ${ }^{7,8}$ but to our knowledge local steroid-induced simultaneous bilateral patellar tendon rupture has not previously been described.

The use of local steroids may have reduced the normally substantial tensile strength of the tendon 


\section{S.C. Clark et al.}

to the extent where linear tension on falling caused simultaneous rupture through the substance. It has been suggested that steroids injected locally may have this effect, although there has been conflicting evidence on the direct effects of steroids on tendons. ${ }^{9}$

Local steroid injections into the patellar tendon for symptomatic relief of sports injuries may have significant sequelae in the event of tendon rupture. Great care must be taken not to penetrate the substance of the patellar tendon to avoid the potential outcome of tendon rupture. The consequences of steroid administration around a tendon are as yet undetermined.

\section{REFERENCES}

1. McMaster P.E. (1933) Tendon and muscle ruptures. Journal of Bone and Surgery 15, 705.

2. Giblin P., Small A. \& Nichol R. (1982) Bilateral rupture of the ligamentum patellae: two case reports and a review of the literature. Australian and New Zealand
Journal of Surgery 54, 145.

3. Razzano C.D., Wilde A.H. \& Phalen G.S. (1973) Bilateral rupture of the infrapatella tendon in rheumatoid arthritis. Clinical Orthopedics 91, 58.

4. Strejeck J. \& Popelka S. (1969) Bilateral rupture of the patella ligaments in systemic lupus erythematosus. Lancet 2, 743.

5. Preston E.T. (1972) Avulsion of both quadriceps tendons in hypothyroidism. Journal of the American Medical Association 221, 406.

6. Costigan P.S. \& Innes A. (1992) Spontaneous bilateral rupture of the quadriceps mechanism in chronic renal failure. Journal of the Royal College of Surgeons of Edinburgh 37, 343.

7. Ismail A.M., Balakrishnan R. \& Rajakumar M.K. (1969) Rupture of patellar ligament after steroid infiltration. Journal of Bone and Joint Surgery 3, 503.

8. Unvesferth L.J. \& Olix M.L. (1973) The effect of local steroid injections on tendon Journal of Sports Medicine 1, 31.

9. Wrenn R.N., Goldner J.L. \& Markee J.L. (1954) An experimental study of the effect of cortisone on the healing process and tensile strength of tendons. Journal of Bone and Joint Surgery 36A, 588. 